\title{
Definitions of local density in density-dependent potentials for mixtures
}

\author{
Peter Vanya $\odot^{1,2, *}$ and James A. Elliott $\odot^{1, \dagger}$ \\ ${ }^{1}$ Department of Materials Science and Metallurgy, University of Cambridge, 27 Charles Babbage Road, \\ Cambridge CB3 OFS, United Kingdom \\ ${ }^{2}$ Value for Money Unit, Ministry of Finance of the Slovak Republic, Štefanovičova 5, 81782 Bratislava, Slovakia
}

(Received 19 September 2019; revised 10 May 2020; accepted 2 July 2020; published 30 July 2020)

\begin{abstract}
Density-dependent potentials are frequently used in materials simulations because of their approximate description of many-body effects at minimal computational cost. However, in order to apply such models to multicomponent systems, an appropriate definition of total local particle density is required. Here, we discuss two definitions of local density in the context of many-body dissipative particle dynamics. We show that only a potential which combines local densities from all particle types in its argument gives physically meaningful results for all composition ratios. Drawing on the ideas from metal potentials, we redefine local density such that it can accommodate different intertype interactions despite the constraint to keep the main interaction parameter constant, known as Warren's no-go theorem, and generalize the many-body potential to heterogeneous systems. We then show via simulation how liquid-liquid and liquid-solid coexistence can arise just by tuning the interaction parameters.
\end{abstract}

DOI: 10.1103/PhysRevE.102.013312

\section{INTRODUCTION}

Coarse graining is a widely used approach across all scales to eliminate fast or unimportant degrees of freedom, speed up simulations, and understand the most relevant physics. In electronic structure theory, core electrons are often coarse-grained into pseudopotentials; in classical atomistic simulations, all electrons are reduced to effective potentials between atoms; in soft matter, whole atoms and molecules can be collapsed into particles. These "blobs" interact via effective pair potentials, which differ from classical, all-atom pair potentials in that they are parametrized only for a specific thermodynamic state. Thus, these potentials do not necessarily reproduce material properties at temperatures or pressures other than the one for which they were defined, which is commonly known as the transferability problem.

A dependence on the local density (LD) of particles can be added to increase predictive abilities, motivated by the observation that some material properties cannot be captured purely by pair potentials $[1,2]$. In the solid state, $L D$ is used to improve the description of metals and alloys. Coarse graining electrons into an effective local electronic density significantly improves the description of fracture and the role of impurities. Examples of such metal potentials are the embedded atom method [3,4], Finnis-Sinclair model [5], and Sutton-Chen potential [6].

The general form of the energy in these metal potentials is

$$
U=\sum_{i<j} V\left(r_{i j}\right)+\sum_{i} u\left(\bar{\rho}_{i}\right)
$$

\footnotetext{
*peter.vanya@gmail.com

†jae1001@cam.ac.uk
}

where $u\left(\bar{\rho}_{i}\right)$ is the self-energy of $i$ th atom embedded in a local density $\bar{\rho}_{i}$ and $r_{i j}=\left|\mathbf{r}_{i}-\mathbf{r}_{j}\right|$ is the distance between the $i$ th and $j$ th atoms at positions $\mathbf{r}_{i}$ and $\mathbf{r}_{j}$, respectively. The local density accounts for neighboring particles via weight functions $w_{\rho}$ which decrease with distance:

$$
\bar{\rho}_{i}=\sum_{j \neq i} w_{\rho}\left(r_{i j}\right) .
$$

For the Finnis-Sinclair and Sutton-Chen potentials, $u\left(\bar{\rho}_{i}\right)=$ $-A \sqrt{\bar{\rho}_{i}}$, where the square root is motivated by the tight binding approximation. The force on $i$ th atom is

$$
\mathbf{F}_{i}=-\frac{\partial U}{\partial \mathbf{r}_{i}}=-\sum_{j} \frac{\partial U}{\partial r_{i j}} \hat{\mathbf{r}}_{i j},
$$

where $\hat{\mathbf{r}}_{i j}=\left(\mathbf{r}_{i}-\mathbf{r}_{j}\right) / r_{i j}$ is a unit vector.

In soft matter, potentials with LD terms have been either used with a form defined a priori in many-body dissipative particle dynamics (MDPD) or coarse-grained from the bottom up, as with an application to mixing of water and benzene $[7,8]$. Building on standard dissipative particle dynamics (DPD) [9], MDPD is suitable for describing mesoscale systems with heterogeneous densities. This force field was introduced by Pagonabarraga and Frenkel [10] in general terms as a potential for nonideal fluids and further developed by Trofimov et al. [11] and Warren [12,13]. MDPD was recently parametrized by the present authors for solvent mixtures [14].

In past decades, a significant body of research has been generated by applying standard or many-body DPD. The Karniadakis group investigated a range of applications including blood [15-17], block copolymers [18], and membranes [19]. Ghoufi and Malfreyt explored extensively the vapor-liquid coexistence in MDPD [20-22]. Merabia et al. investigated wetting of liquid on a solid substrate with a model similar to the present definition of MDPD [23-25]. Another, related 
branch of research is smoothed-particle hydrodynamics, accounting for thermal fluctuations and transport by discretizing the Navier-Stokes equations [26-29].

Standard DPD has a purely repulsive pair potential with a cutoff yielding a force between two coarse-grained particles of the form

$$
\mathbf{F}_{i j}=A w\left(r_{i j}\right) \hat{\mathbf{r}}_{i j},
$$

where $A$ is an interaction parameter and $w(r)$ a weight function with a linear taper: $w(r)=1-r / r_{\mathrm{c}}$ for $r<r_{\mathrm{c}}$ and zero elsewhere. Together with the many-body term with selfenergy $u(\bar{\rho})$, it can be shown via Eq. (3) that the force from the MDPD potential is

$$
\mathbf{F}_{i j}=A w\left(r_{i j}\right) \hat{\mathbf{r}}_{i j}-\left[u^{\prime}\left(\bar{\rho}_{i}\right)+u^{\prime}\left(\bar{\rho}_{j}\right)\right] w_{\rho}^{\prime}\left(r_{i j}\right) \hat{\mathbf{r}}_{i j} .
$$

This weight function has a cutoff and is normalized, such that $\int 4 \pi r^{2} w_{\rho}(r) d r=1$.

The simplest form of self-energy is $u\left(r_{i}\right)=B \bar{\rho}_{i}^{2} / 2$, with an interaction parameter $B$ and $w_{\rho}(r) \sim\left(1-r / r_{\mathrm{d}}\right)^{2}$ for $r<r_{\mathrm{d}}$ and zero elsewhere. ${ }^{1}$ Setting $A<0, B>0$ and the many-body cutoff $r_{\mathrm{d}}<r_{\mathrm{c}}$ results in a potential that can produce a liquidvapor coexistence, which makes MDPD applicable to systems containing interfaces between different phases.

\section{A. Multicomponent systems}

The generalization of self-energy in MDPD to multicomponent systems has so far been ambiguous; the form of the LD proposed in the literature has been assumed without justification of the reasoning. For the $i$ th particle, single-component LDs can be defined separately by particle type $\alpha$ :

$$
\bar{\rho}_{i}^{(\alpha)}=\sum_{j, j \in \alpha} w_{\rho}\left(r_{i j}\right)
$$

So, for $n$-component systems there are $n$ different LDs for each particle. The ambiguity lies in the fact that it is not $a$ priori clear how to combine these correctly in the self-energy.

Mathematically, for pair potentials the energy per particle is a straightforward function of the coordinates of the neighboring particles: $u\left(\left\{\mathbf{r}_{i}\right\}\right)$. LD potentials have a function representing the LD and an outside wrapping function $u$ : $u\left[\bar{\rho}\left(\left\{\mathbf{r}_{i}\right\}\right)\right]$. In the case of multicomponent systems, such as liquid mixtures or alloys, a question arises how to combine the terms within the wrapping function $u(x)$. Taking the simplest, two-component system composed of types $\alpha$ and $\beta$, two options are immediately apparent: (i) $u\left[\bar{\rho}^{(\alpha)}\left(\left\{\mathbf{r}_{i}\right\}\right)+\bar{\rho}^{(\beta)}\left(\left\{\mathbf{r}_{i}\right\}\right)\right]$ and (ii) $u\left[\bar{\rho}^{(\alpha)}\left(\left\{\mathbf{r}_{i}\right\}\right)\right]+u\left[\bar{\rho}^{(\beta)}\left(\left\{\mathbf{r}_{i}\right\}\right)\right] .^{2}$ We denote these as the partial and full LD variants respectively.

The partial LD variant was used by Sanyal et al. [8] and also had originally been implemented in the DL_MESO package [30], which has inspired the present exploration. The

\footnotetext{
${ }^{1}$ In general, any power of the local density can be considered and thus the equation of state can be influenced, as was demonstrated by Trofimov et al. [11].

${ }^{2}$ There is also a trivial function where the self-energy contains only the local density of the particles of the like type, $u\left[\bar{\rho}^{(\alpha)}\left(\mathbf{r}_{i}\right)\right]$. However, the resulting force is the same as for the partial local density variant.
}



FIG. 1. A minimal multicomponent system to explore local density potentials.

total LD variant was discussed in Sec. V of the work by Trofimov et al. [11] and implicitly by Warren [31], when introducing the no-go theorem stating that the parameter $B$ must be constant across particle types if the potential $U$ is to be conservative.

In this work, we discuss the viability of these two LD variants of multicomponent systems. We show that the partial variant can have type-dependent parameters $B^{(\alpha \beta)}$, but it behaves unphysically in that a simple relabelling of particles alters the forces between them. This in turn affects local ordering and phase behavior. As a result, only the total LD variant is usable in practice. Drawing on research in solid-state physics, we redefine the latter such that it can accommodate variability among different particle types.

To illustrate these points, we take a minimal twocomponent mixture of three particles and explicitly compute the forces between them for each of the variants of local density (Fig. 1). We work with a general form of self-energy $u\left(\bar{\rho}_{i}\right)$ and weight function $w_{\rho}(r)$ and assume that all the particles are within the cutoff distance of one another. This reasoning also applies to other density-dependent potentials, not just MDPD.

To proceed with algebra, we first define the necessary notation. In the most general case, there are three different interaction parameters in a two-component system. Like particles of types $\alpha$ and $\beta$ interact via parameters $B^{(\alpha \alpha)}$ and $B^{(\beta \beta)}$, respectively, and unlike particles via $B^{(\alpha \beta)}$. To treat the interaction parameter $B$ as an explicit prefactor, as in the case of metal potentials, we introduce a wrapping function $\psi$ such that $u\left(\bar{\rho}_{i}\right)=B \psi\left(\bar{\rho}_{i}\right)$. Commonly, $\psi(\bar{\rho})$ is a polynomial, $\psi=\bar{\rho}^{n} / n$, with $n=2$ in MDPD.

\section{DERIVATION OF FORCES FOR PARTIAL LOCAL DENSITIES}

Starting with the partial LD variant, the local densities can be listed explicitly (Table I) for each of the three particles in the minimal system. From these, the self-energies of the particles follow

$$
\begin{aligned}
& u_{1}=B^{(\alpha \alpha)} \psi\left(\bar{\rho}_{1}^{(\alpha)}\right)+B^{(\alpha \beta)} \psi\left(\bar{\rho}_{1}^{(\beta)}\right), \\
& u_{2}=B^{(\alpha \alpha)} \psi\left(\bar{\rho}_{2}^{(\alpha)}\right)+B^{(\alpha \beta)} \psi\left(\bar{\rho}_{2}^{(\beta)}\right), \\
& u_{3}=B^{(\alpha \beta)} \psi\left(\bar{\rho}_{3}^{(\alpha)}\right)+B^{(\beta \beta)} \psi\left(\bar{\rho}_{3}^{(\beta)}\right) .
\end{aligned}
$$

The total energy $U=u_{1}+u_{2}+u_{3}$. Computing, e.g., $\partial U / \partial \mathbf{r}_{1}$,

$$
\begin{aligned}
\frac{\partial U}{\partial \mathbf{r}_{1}}= & B^{(\alpha \alpha)}\left[\psi^{\prime}\left(\bar{\rho}_{1}^{(\alpha)}\right)+\psi^{\prime}\left(\bar{\rho}_{2}^{(\alpha)}\right)\right] w_{\rho}^{\prime}\left(r_{12}\right) \hat{\mathbf{r}}_{12} \\
& +B^{(\alpha \beta)}\left[\psi^{\prime}\left(\bar{\rho}_{1}^{(\beta)}\right)+\psi^{\prime}\left(\bar{\rho}_{3}^{(\alpha)}\right)\right] w_{\rho}^{\prime}\left(r_{13}\right) \hat{\mathbf{r}}_{13},
\end{aligned}
$$


TABLE I. List of partial local densities for each particle.

\begin{tabular}{llll}
\hline \hline Particle & \multicolumn{1}{c}{ Partial, type $\alpha$} & Partial, type $\beta$ & \multicolumn{1}{c}{ Total } \\
\hline 1 & $\bar{\rho}_{1}^{(\alpha)}=w_{\rho}\left(r_{12}\right)$ & $\bar{\rho}_{1}^{(\beta)}=w_{\rho}\left(r_{13}\right)$ & $\bar{\rho}_{1}=w_{\rho}\left(r_{12}\right)+w_{\rho}\left(r_{13}\right)$ \\
2 & $\bar{\rho}_{2}^{(\alpha)}=w_{\rho}\left(r_{12}\right)$ & $\bar{\rho}_{2}^{(\beta)}=w_{\rho}\left(r_{23}\right)$ & $\bar{\rho}_{2}=w_{\rho}\left(r_{12}\right)+w_{\rho}\left(r_{23}\right)$ \\
3 & $\bar{\rho}_{3}^{(\alpha)}=w_{\rho}\left(r_{13}\right)+w_{\rho}\left(r_{23}\right)$ & $\bar{\rho}_{3}^{(\beta)}=0$ & $\bar{\rho}_{3}=w_{\rho}\left(r_{13}\right)+w_{\rho}\left(r_{23}\right)$ \\
\hline \hline
\end{tabular}

$\partial U / \partial \mathbf{r}_{2}$ can be obtained from Eq. (10) by simply transposing particle indices 1 and 2 and

$$
\begin{aligned}
\frac{\partial U}{\partial \mathbf{r}_{3}}= & B^{(\alpha \beta)}\left[\psi\left(\bar{\rho}_{1}^{(\beta)}\right)+\psi^{\prime}\left(\bar{\rho}_{3}^{(\alpha)}\right)\right] w_{\rho}^{\prime}\left(r_{12}\right) \hat{\mathbf{r}}_{31} \\
& +B^{(\alpha \beta)}\left[\psi^{\prime}\left(\bar{\rho}_{2}^{(\beta)}\right)+\psi\left(\bar{\rho}_{3}^{(\alpha)}\right)\right] w_{\rho}^{\prime}\left(r_{23}\right) \hat{\mathbf{r}}_{32} .
\end{aligned}
$$

Every force $\mathbf{F}_{i j}$ has the form of Eq. (3) and $\mathbf{F}_{i j}=-\mathbf{F}_{j i}$ for every pair $\{i, j\}$ in line with Newton's third law. Hence, the self-energy of the partial LD variant is conservative and, at the same time, allows for type-specific interaction parameters $B$.

\section{PROBLEM WITH PARTICLE RELABELING}

The freedom to use different parameters for unlike types provided by the partial LD variant can be important for an appropriate depiction of phase behavior of mixtures. However, a new problem arises: The interaction strength of particles of unlike types is artificially lowered only because they have different labels, not because of physical differences.

In a homogeneous, single-component MDPD liquid with parameter $B$, the local density is same for every particle and equal to the global density, $\bar{\rho}_{i} \approx \rho$ (assuming mean-field approximation). The force between any two particles is then

$$
\begin{aligned}
\mathbf{F}_{i j} & \approx B\left[\psi^{\prime}(\rho)+\psi^{\prime}(\rho)\right] w_{\rho}^{\prime}\left(r_{i j}\right) \hat{\mathbf{r}}_{i j} \\
& =2 B \psi^{\prime}(\rho) w_{\rho}\left(r_{i j}\right) \hat{\mathbf{r}}_{i j} .
\end{aligned}
$$

Consider randomly splitting all the particles into two types but keeping the interaction parameter constant, $B^{(\alpha \alpha)}=$ $B^{(\alpha \beta)}=B^{(\beta \beta)}=B$. Now, every particle sees around itself, on average, one-half of the particles of type $\alpha$ and the other half of type $\beta$, as the system remains physically the same and hence perfectly mixed. Hence, the average local density of

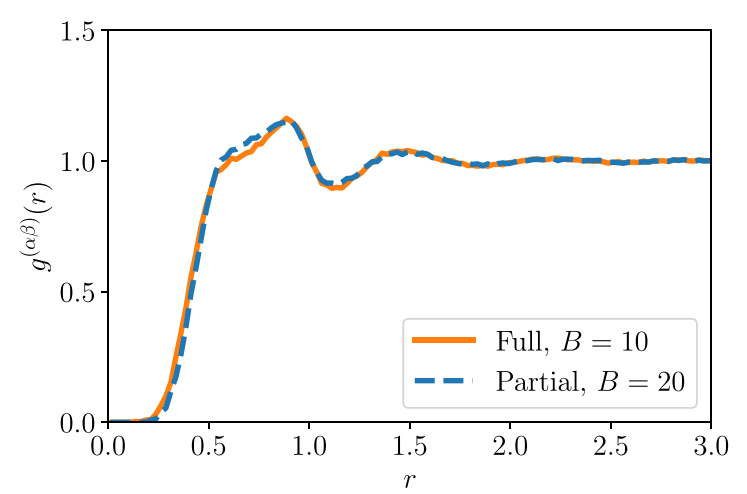

FIG. 2. Intertype radial distribution functions of two variants of local density demonstrating the unphysical behavior of the partial variant. The slight deviation is most likely due to the different temperatures at which these systems equilibrate. both type $\alpha$ and $\beta$ particles is $\bar{\rho}_{i}^{(\alpha / \beta)} \approx \rho / 2$. Computing the force between like type particles $i$ and $j$ yields

$$
\begin{aligned}
\mathbf{F}_{i j} & =B\left[\psi^{\prime}(\rho / 2)+\psi^{\prime}(\rho / 2)\right] w_{\rho}^{\prime}\left(r_{i j}\right) \hat{\mathbf{r}}_{i j} \\
& =2 B \psi^{\prime}(\rho / 2) w_{\rho}\left(r_{i j}\right) \hat{\mathbf{r}}_{i j} .
\end{aligned}
$$

These forces are not equal, since generally $\psi^{\prime}(\rho) \neq \psi^{\prime}(\rho / 2)$. The only exception is the case when $\psi$ depends linearly on $\bar{\rho}$ and $\psi^{\prime} \sim 1$, which is the force field of standard DPD. With the simplest nontrivial definition of self-energy, $\psi(\bar{\rho})=\bar{\rho}^{2} / 2$, the force on any particle would become twice as small purely due to relabeling, and, in simulations of mixtures with $m \gg 1$ components, $m$ times smaller.

This flaw is manifest in the local structure of a liquid, which is represented by the radial distribution function (RDF). Using DL_MESO version 2.6 [30], we set up two simulations of an effectively single-component liquid with arbitrarily relabeled particles, exploring the full $\mathrm{LD}$ variant with parameter $B=10$ and partial LD variant with $B=20$, which should be different liquids. In each case, we measured $g^{(\alpha \beta)}(r)$. Figure 2 shows the near identity of these two RDFs, demonstrating that the partial LD variant artificially lowers the interaction by a factor of 2 .

To illustrate the practical consequences of this, we consider a binary liquid interacting via an MDPD potential where interaction parameters are constant across particle types, $A=-18$ and $B=8$. These values represent water at a coarse-graining degree 6 with equilibrium density $\rho_{0}=6.70$ [32]. Setting the density to $\rho=7>\rho_{0}$, this effectively single-component liquid with arbitrarily relabeled particles should homogeneously fill the simulation cell. We simulated the two local density variants and composed density profiles for each type after equilibration. Figure 3 shows that the expected phase behavior



(a)

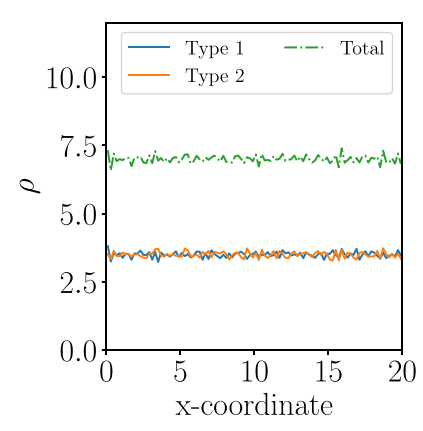

(b)
FIG. 3. Density profiles of a homogeneous liquid with particles randomly split into two types, for (a) partial and (b) total local density variants. The former produces an unphysically high global density and a spurious vapor phase. The values of $\rho$ and $x$ are given in DPD units. 
for a homogeneous liquid is only reproduced with the total LD variant.

\section{GENERALIZED MANY-BODY FORCE FIELD}

\section{A. Derivation of forces for total local densities}

Having shown that the partial LD variant produces unphysical behavior, only the total LD variant, which works with $\bar{\rho}_{i}=\bar{\rho}_{i}^{(\alpha)}+\bar{\rho}_{i}^{(\beta)}$, remains. However, Warren's no-go theorem states that, for a type-independent definition of local density [Eq. (6)], only a type-independent parameter $B$ is allowed, i.e., $u_{i}=B \psi\left(\bar{\rho}_{i}\right) \forall i$ [31]. This means that the self-energy for the force on the, e.g., first particle with the following form:

$$
\begin{aligned}
\mathbf{F}_{1}= & -B^{(\alpha \alpha)}\left(\psi^{\prime}\left(\bar{\rho}_{1}\right)+\psi^{\prime}\left(\bar{\rho}_{2}\right)\right) w_{\rho}^{\prime}\left(r_{12}\right) \hat{\mathbf{r}}_{12} \\
& -B^{(\alpha \beta)}\left(\psi^{\prime}\left(\bar{\rho}_{1}\right)+\psi^{\prime}\left(\bar{\rho}_{3}\right)\right) w_{\rho}^{\prime}\left(r_{13}\right) \hat{\mathbf{r}}_{13}
\end{aligned}
$$

does not exist unless $B^{(\alpha \alpha)}=B^{(\alpha \beta)}=B^{(\beta \beta)}$. This constraint on $B$ means that it is not possible to distinguish particles in MDPD purely by the many-body potential term, which limits the versatility of this method.

Borrowing from the formalism of metal potentials, we show that there is a way to preserve type-dependent forces by introducing a type-dependent local density [33,34]. Generally the local density for particle of type $\alpha$ accounting for neighboring particles of type $\beta$ is

$$
\bar{\rho}_{i}^{(\alpha \beta)}=\sum_{j} w_{\rho}^{(\alpha \beta)}\left(r_{i j}\right), \quad i \in \alpha, \quad j \in \beta .
$$

For a two-component system, there are four possible local densities: $\bar{\rho}^{(\alpha \alpha)}, \bar{\rho}^{(\alpha \beta)}, \bar{\rho}^{(\beta \alpha)}, \bar{\rho}^{(\beta \beta)}$. Generally, the influence of a particle of type $\alpha$ to the local density of a particle of type $\beta$ might not be the same, but in practice $\bar{\rho}^{(\alpha \beta)}=\bar{\rho}^{(\beta \alpha)}$.

Considering again the minimal three-particle system (Fig. 1), the self-energies are

$$
\begin{aligned}
& u_{1}=B \psi\left(\bar{\rho}_{1}^{(\alpha \alpha)}+\bar{\rho}_{1}^{(\alpha \beta)}\right)=B \psi\left(\bar{\rho}_{1}\right), \\
& u_{2}=B \psi\left(\bar{\rho}_{2}^{(\alpha \alpha)}+\bar{\rho}_{2}^{(\alpha \beta)}\right)=B \psi\left(\bar{\rho}_{2}\right), \\
& u_{3}=B \psi\left(\bar{\rho}_{3}^{(\alpha \beta)}+\bar{\rho}_{3}^{(\beta \beta)}\right)=B \psi\left(\bar{\rho}_{3}\right),
\end{aligned}
$$

and the total energy $U=u_{1}+u_{2}+u_{3}$. Differentiating to obtain the force on particle 1 yields

$$
\begin{aligned}
\mathbf{F}_{1}= & -B\left[\psi^{\prime}\left(\bar{\rho}_{1}\right)+\psi^{\prime}\left(\bar{\rho}_{2}\right)\right] w_{\rho}^{\prime(\alpha \alpha)}\left(r_{12}\right) \hat{\mathbf{r}}_{12} \\
& -B\left[\psi^{\prime}\left(\bar{\rho}_{1}\right)+\psi^{\prime}\left(\bar{\rho}_{3}\right)\right] w_{\rho}^{\prime(\alpha \beta)}\left(r_{13}\right) \hat{\mathbf{r}}_{13} \\
= & -\mathbf{F}_{12}-\mathbf{F}_{13},
\end{aligned}
$$

and the forces on forces on particles 2 and 3 can be obtained similarly.

The cross-type interaction is now represented by the term $B w_{\rho}^{\prime(\alpha \beta)}$, which gives the freedom to tune it via the derivative of the weight function $w_{\rho}^{(\alpha \beta)}$. A simple arithmetic mixing rule can be used, e.g. $w_{\rho}^{(\alpha \beta)}=\left(w_{\rho}^{(\alpha \alpha)}+w_{\rho}^{(\beta \beta)}\right) / 2$, but a more general form might be required for coarse-grained systems, possibly involving the Flory-Huggins $\chi$ parameter as in the case of standard DPD. (A geometric mixing, as in the case of metal potentials [33], might not be suitable due to the explicit and necessary cutoff of coarse-grained potentials.)

\section{B. Mixing of different material types}

Finally, we briefly address the mixing of different materials. An example is a liquid or a polymer on metal surface, which is a typical setting for heterogeneous catalysis and so is of immense practical importance.

The total LD self-energy allows for definitions of the wrapping function $\psi$ depending on the material phase. Referring to Fig. 1, consider the first two particles metallic (M) and the third a liquid (L). Each phase has a different wrapping function: $\psi_{\mathrm{M}}$ for a metal can be a square root, and $\psi_{\mathrm{L}}$ for a liquid can be a square to allow for liquid-vapor coexistence. The self-energies are

$$
\begin{aligned}
& u_{1}=B \psi_{\mathrm{M}}\left(\bar{\rho}_{1}^{(\alpha \alpha)}+\bar{\rho}_{1}^{(\alpha \beta)}\right)=B \psi_{\mathrm{M}}\left(\bar{\rho}_{1}\right), \\
& u_{2}=B \psi_{\mathrm{M}}\left(\bar{\rho}_{2}^{(\alpha \alpha)}+\bar{\rho}_{2}^{(\alpha \beta)}\right)=B \psi_{\mathrm{M}}\left(\bar{\rho}_{2}\right), \\
& u_{3}=B \psi_{\mathrm{L}}\left(\bar{\rho}_{3}^{(\alpha \beta)}+\bar{\rho}_{3}^{(\beta \beta)}\right)=B \psi_{\mathrm{L}}\left(\bar{\rho}_{3}\right) .
\end{aligned}
$$

Following the previous section, the force on particle 1 is

$$
\begin{aligned}
\mathbf{F}_{1}= & -B\left[\psi_{\mathrm{M}}^{\prime}\left(\bar{\rho}_{1}\right)+\psi_{\mathrm{M}}^{\prime}\left(\bar{\rho}_{2}\right)\right] w_{\rho}^{\prime(\alpha \alpha)}\left(r_{12}\right) \hat{\mathbf{r}}_{12} \\
& -B\left[\psi_{\mathrm{M}}^{\prime}\left(\bar{\rho}_{1}\right)+\psi_{\mathrm{L}}^{\prime}\left(\bar{\rho}_{3}\right)\right] w_{\rho}^{\prime(\alpha \beta)}\left(r_{13}\right) \hat{\mathbf{r}}_{13},
\end{aligned}
$$

where $\mathbf{F}_{13}$ contains one term from neighboring metal atoms, $\psi_{\mathrm{M}}^{\prime}$, and another from neighboring liquid particles, $\psi_{\mathrm{L}}^{\prime}$. The cross-type weight function $w_{\rho}^{(\alpha \beta)}$ requires further investigation to determine its appropriate form or a range of forms, but at this stage it is sufficient to note that, as before, it represents unambiguously the material-dependent interaction via the term $B w_{\rho}^{\prime(\alpha \beta)}$.

\section{Exploring new force fields via simulation}

To demonstrate the versatility of this generalized MDPD force field for describing inhomogeneous mixtures, we perform several simulations using a custom-written code [35].

Before doing so, it is necessary to redefine the repulsion parameter $B$ to follow the logic of the embedded atom method using unique $B$ 's but varying parameters within the wrapping functions with particle type. The convention with MDPD has been so far to define $B$ through the force $F_{i j}=$ $B_{\mathrm{o}}\left(\bar{\rho}_{i}+\bar{\rho}_{j}\right)\left(1-r / r_{\mathrm{d}}\right)$ with $r<r_{\mathrm{d}}$ [13]. Here, we start from the energy, $u_{i}=B \bar{\rho}_{i}^{2} / 2$, so $B$ is modified by the normalization factor of the weight function; the force for like-type particles then becomes $F_{i j}=15 /\left(\pi r_{\mathrm{d}}^{4}\right) B\left(\bar{\rho}_{i}+\bar{\rho}_{j}\right)\left(1-r / r_{\mathrm{d}}\right)$, so $B=$ $B_{\mathrm{o}} \pi r_{\mathrm{d}}^{4} / 15$. For a typical value of $B_{\mathrm{o}}=30, B \approx 2$ for $r_{\mathrm{d}}=$ 0.75 .

Using this convention, we first investigate inhomogeneous liquids with same quadratic wrapping function for both particle types, $\psi(x)=x^{2} / 2$, with parameters $A=-40, B=2$. The cross-type repulsion is represented by decreased attraction $A_{12}=A+\Delta A$, setting $\Delta A=20$. The difference between the liquids is marked by the many-body cutoff set to 0.75 and 0.60 for type 1 and type 2, respectively, and cross interaction as the arithmetic mean of these, 0.675. The simulation contained 2000 particles, split equally between the two types, in a $20 \times 5 \times 5$ cell and was run for 200 reduced time units with time step $\Delta t=0.01$. Figure 4 shows a coexistence of two separated phases at different densities, a feature so far unavailable in MDPD. 


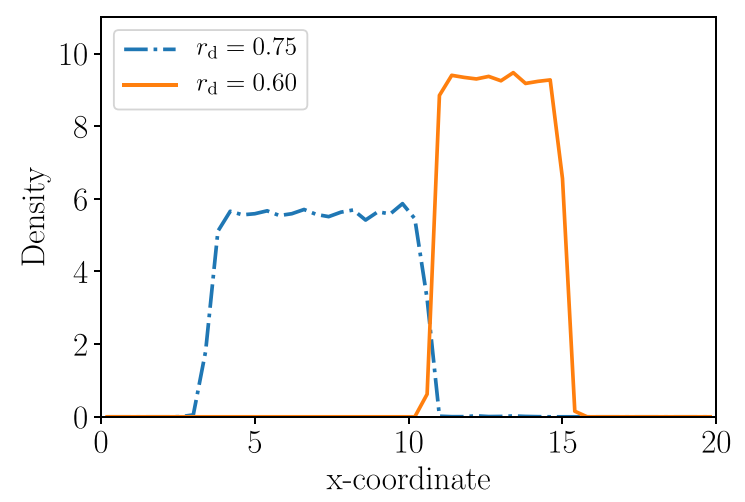

FIG. 4. A coexistence of inhomogeneous liquids using a generalized MDPD force field allowing for different many-body cutoffs.

As a second simulation, following Eq. (22), we consider a system with wrapping functions differing with particle types. For simplicity, we constrain the choice to polynomial functions: $\psi(x)=x^{N_{\mathrm{w}}} / N_{\mathrm{w}}$. We chose $N_{\mathrm{w}}=2$ for phase 1 and $N_{\mathrm{w}}=3$ for phase 2 , the repulsion parameter $B=3$, and manybody cutoffs $r_{\mathrm{d}}^{(11)}=0.75, r_{\mathrm{d}}^{(22)}=0.55$, and $r_{\mathrm{d}}^{(12)}=0.65$. We simulated for the same time period as before using a smaller time step, $\Delta t=0.006$ in order to retain the temperature within $10 \%$ of $k_{\mathrm{B}} T=1$. This setup shows the coexistence of a liquid phase and a solid phase (Fig. 5). The solid phase is identified by a radial distribution function with a sharp first peak and more frequent oscillations, in contrast to that for the liquid phase, which is flatter and smoother (in the inset of Fig. 5). This can be compared with Fig. 5(a) of our previous work [14].

With these exploratory simulations, we note several observations. First, it is not advisable to use $N_{\mathrm{w}}=1 / 2$, as in the embedded atom method, due to the finiteness of the MDPD potential. This feature produces, with some non-negligible probability, vanishing local densities, from which, after taking the derivative to compute forces, results a zero in the denominator. A solution could be to increase the range, but this would go against the spirit of coarse graining and simulation efficiency. Second, with increasing $N_{\mathrm{w}}$ it is necessary to minimize the box prior to the simulation run; such a need does not arise with lower values due to fast equilibration. Third, it is also possible to achieve a liquid-solid phase coexistence using purely $N_{\mathrm{w}}=2$ for both particle types and controlling the difference between the phases only by the many-body cutoff. Fourth, we only set the cross-type repulsion via the interaction parameter $A$ and many-body cutoff $r_{\mathrm{d}}$; there are other options to modify the many-body part of the interaction apart from the parameter $r_{\mathrm{d}}$ that could provide further flexibility to describe real materials; these remain to be investigated.

Finally, there is the question of applications of the generalized MDPD force field to real materials. Standard DPD as well as many-body DPD relied on ad hoc parametrization

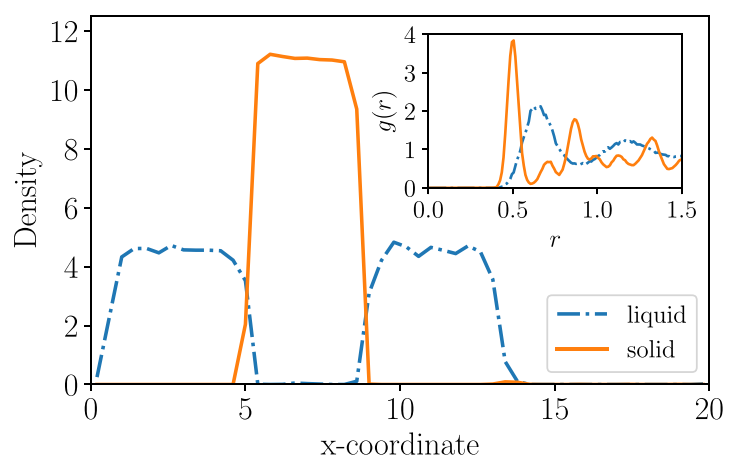

FIG. 5. A coexistence of liquid and solid phase using a generalized MDPD force field achieved by choosing different many-body cutoffs and wrapping functions. The inset shows radial distribution functions; the tall first peak of the orange curve is a signature of the solid phase.

dependent on the choice of the material by setting the reduced length scale based on its molecular volume. However, each component of a heterogeneous mixture has its own scale, so a new parametrization protocol must be devised. As the atomistic resolution, on which the EAM is based, is not available, and the metallic faces and lattice constants are ambiguous concepts in coarse graining, an open question remains about what the default physical properties for the parametrization of the solid state should be. It could be possible to use the total energy and compressibility.

\section{CONCLUSION}

The definition of local density for many-body, densitydependent potentials must include all particle types. Otherwise, a mathematical relabeling of particles yields unphysical results unless the potential energy is redefined. Following the idea of alloys in metal potentials, we showed that the total local density composed of contributions from all particles can be distinguished by the particle type and is able to account for different cross-type interactions despite the need to keep the main interaction parameter constant to obey Warren's no-go theorem [31].

We generalized the many-body coarse-grained potential and demonstrated its usefulness via simulations of a mixture of liquids with unequal densities and a liquid-solid system. This force field thus opens up avenues for treatment of material combinations such as soft matter on metal surface with potential applications in heterogeneous catalysis.

\section{ACKNOWLEDGMENTS}

The authors thank Johnson Matthey and the Engineering and Physical Sciences Research Council (EPSRC) for financial support and Soňa Slobodníková for inspiring scientific discussions.
[1] A. A. Louis, J. Phys.: Condens. Matter 14, 9187 (2002).

[2] S. Merabia and I. Pagonabarraga, J. Chem. Phys. 127, 054903 (2007).

[3] M. S. Daw and M. I. Baskes, Phys. Rev. Lett. 50, 1285 (1983).
[4] M. S. Daw and M. I. Baskes, Phys. Rev. B 29, 6443 (1984).

[5] M. W. Finnis and J. E. Sinclair, Philos. Mag. A 50, 45 (1984).

[6] A. P. Sutton and J. Chen, Philos. Mag. Lett. 61, 139 (1990).

[7] T. Sanyal and M. S. Shell, J. Chem. Phys. 145, 034109 (2016). 
[8] T. Sanyal and M. S. Shell, J. Phys. Chem. B 122, 5678 (2018).

[9] P. Español and P. B. Warren, J. Chem. Phys. 146, 150901 (2017).

[10] I. Pagonabarraga and D. Frenkel, J. Chem. Phys. 115, 5015 (2001).

[11] S. Y. Trofimov, E. L. F. Nies, and M. a. J. Michels, J. Chem. Phys. 117, 9383 (2002).

[12] P. B. Warren, Phys. Rev. Lett. 87, 225702 (2001).

[13] P. B. Warren, Phys. Rev. E 68, 066702 (2003).

[14] P. Vanya, P. Crout, J. Sharman, and J. A. Elliott, Phys. Rev. E 98, 033310 (2018).

[15] H.-Y. Chang, X. Li, H. Li, and G. E. Karniadakis, PLoS Comput. Biol. 12, e1005173 (2016).

[16] X. Li, Z. Peng, H. Lei, M. Dao, and G. E. Karniadakis, Philos. Trans. R. Soc., A 372, 20130389 (2014).

[17] Z. Peng, X. Li, I. V. Pivkin, M. Dao, G. E. Karniadakis, and S. Suresh, Proc. Natl. Acad. Sci. USA 110, 13356 (2013).

[18] X. Li, I. V. Pivkin, H. Liang, and G. E. Karniadakis, Macromolecules 42, 3195 (2009).

[19] Y. Li, X. Li, Z. Li, and H. Gao, Nanoscale 4, 3768 (2012).

[20] A. Ghoufi and P. Malfreyt, Phys. Rev. E 82, 016706 (2010).

[21] A. Ghoufi and P. Malfreyt, Phys. Rev. E 83, 051601 (2011).
[22] A. Ghoufi, J. Emile, and P. Malfreyt, Eur. Phys. J. E 36, 10 (2013).

[23] S. Merabia and I. Pagonabarraga, Eur. Phys. J. E 20, 209 (2006).

[24] S. Merabia, J. Bonet-Avalos, and I. Pagonabarraga, J. NonNewtonian Fluid Mech. 154, 13 (2008).

[25] S. Merabia and J. B. Avalos, Phys. Rev. Lett. 101, 208304 (2008).

[26] P. Español and M. Revenga, Phys. Rev. E 67, 026705 (2003).

[27] C. Thieulot, L. P. B. M. Janssen, and P. Español, Phys. Rev. E 72, 016713 (2005).

[28] C. Thieulot, L. P. B. M. Janssen, and P. Español, Phys. Rev. E 72, 016714 (2005).

[29] S. Litvinov, M. Ellero, X. Hu, and N. A. Adams, Phys. Rev. E 77, 066703 (2008).

[30] M. A. Seaton, R. L. Anderson, S. Metz, and W. Smith, Mol. Simul. 39, 796 (2013).

[31] P. B. Warren, Phys. Rev. E 87, 045303 (2013).

[32] P. Vanya, J. Sharman, and J. A. Elliott, J. Chem. Phys. 150, 064101 (2019).

[33] H. Rafii-Tabar and A. P. Sulton, Philos. Mag. Lett. 63, 217 (1991).

[34] R. A. Johnson, Phys. Rev. B 39, 12554 (1989).

[35] Available at https://github.com/petervanya/DPDsim. 